\title{
TRAJECTORY CORRECTION MANOEUVRES IN THE TRANSFER TO LIBRATION POINT ORBITS
}

\author{
G. GÓMEZ, M. MARCOTE
}

IEEC \& Departament de Matemàtica Aplicada i Anàlisi

Universitat de Barcelona, Gran Via 545, 08007 Barcelona, Spain

\section{J.J. MASDEMONT}

IEEC \& Departament de Matemàtica Aplicada I

Universitat Politècnica de Catalunya, Diagonal 647, 08028 Barcelona, Spain

In this paper we study the manoeuvres to be done by a spacecraft in order to correct the error in the execution of the injection manoeuvre in the transfer trajectory. We will consider the case in which the nominal trajectory is a halo orbit around the collinear equilibrium point $L_{1}$. The results can be easily extended to the $L_{2}$ point and to other kinds of libration point orbits, such as Lissajous and quasi-halo orbits. For our study we use simple dynamical systems concepts related with the invariant manifolds of the target orbit, and we compare our results with those obtained by Serban et al. ${ }^{14}$ using optimal control.

\section{Introduction}

This paper is devoted to the study of the so called Trajectory Correction Manoeuvres (TCM) problem, that deals with the manoeuvres to be done by a spacecraft in the transfer segment between the parking orbit and the target nominal one. The main purpose of the TCMs is to correct the error introduced by the injection manoeuvre in the transfer trajectory due to the 
inaccuracies of the launch vehicle.

In connection with the Genesis mission ( see $^{10}$ ), the TCM problem has been studied in ${ }^{8,14}$. For this mission a halo type orbit, around the $L_{1}$ point of the Earth-Sun system, is used as nominal orbit. Since this orbit has a strong hyperbolic character, following the ideas introduced in ${ }^{4,6}$, it is possible to use its stable manifold for the transfer, avoiding the insertion manoeuvre into the halo orbit. This is what is know in the literature as the dynamical systems approach to the transfer problem. Other approaches use straightforward propagation from Earth launch conditions to find orbits between the Earth and the halo orbits, keeping some boundary conditions and constraints, at the same time that minimise the total fuel consumption during the transfer (see ${ }^{3,7,9,11}$ ). In any case, one of the conclusions of all these studies is that the insertion manoeuvre, from a parking orbit around the Earth to the transfer trajectory, is a large one, with a $\Delta v$ of the order of $3000 \mathrm{~m} / \mathrm{s}$. For the Genesis mission the error in its execution was expected to be about a $0.2 \%$ of $\Delta v$ ( 1 sigma value) and a key point to be studied is how large is the cost of the correction of this error when the execution of the first correction manoeuvre is delayed.

For the purpose of comparison, in the present study we will use for the main parameters the same values used in ${ }^{14}$. More concretely, we will take as reference model for the simulations, the Restricted Three Body (RTBP) Problem with the same value of the mass ratio $\mu=0.3035910 E-05$, so the gravitational effect of the Moon on the transfer trajectory will not be considered ( see $^{4}$ ). We will also use the same launch conditions near the Earth, which are given in table 1 (from Serban et al. ${ }^{14}$ ).

Table 1. Adimensional initial conditions for the reference transfer trajectory.

\begin{tabular}{lllr}
\hline$x_{0}^{\text {nom }}$ & $-1.000035565608365 E+00$ & $\dot{x}_{0}^{\text {nom }}$ & $1.547585875645079 E-01$ \\
$y_{0}^{\text {nom }}$ & $-1.298950527135473 E-05$ & $\dot{y}_{0}^{\text {nom }}$ & $-3.157800035860918 E-01$ \\
$z_{0}^{\text {nom }}$ & $-1.657172577465346 E-05$ & $\dot{z}_{0}^{\text {nom }}$ & $-1.167438053370118 E-01$ \\
\hline
\end{tabular}

Since the target halo orbit is not explicitly given in ${ }^{14}$, we have used one with approximately the same size as the one displayed in the Figures of the paper, this is a halo periodic orbit with normalised $z$-amplitude (see 12 for the definition) $\beta=0.28$ corresponding to initial conditions: $x(0)=$ $-0.9922709412937017, y(0)=0, z(0)=-0.002456251256325228, \dot{x}(0)=0$, $\dot{y}(0)=0.01191138815471799, \dot{z}(0)=0$. It must be noted that the value of 
the Jacobi constant of the halo orbit, $C=3.000771793017166$, and the one of the above initial conditions for the transfer, $C=3.000782265790755$, do not agree. This means, in particular, that the reference transfer trajectory with the initial conditions given in table 1 , is not an orbit in the stable manifold of the halo periodic orbit. Nevertheless, approximately 110 days after launch, the transfer orbit is very close to the halo one and, at that point, a manoeuvre of about $13.5 \mathrm{~m} / \mathrm{s}$ inserts the spacecraft into the halo. Of course, this insertion manoeuvre could be skipped if the reference initial conditions would belong to the stable manifold but, unfortunately, the departure point rarely meets the constraints associated with actual launch conditions.

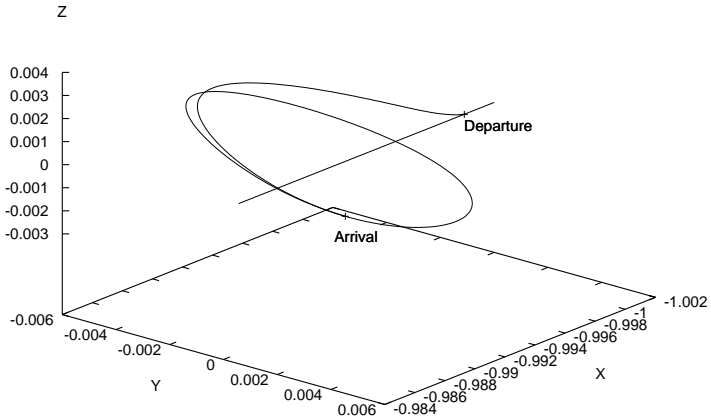

Fig. 1. Reference transfer trajectory and nominal halo orbit, as given in Serban et al., for the study of the TCM problem (adimensional units). The departure and arrival points are separated, approximately 110 days of time of flight.

In Figure 1 we have displayed the solution with the initial conditions given in table 1 as well as the nominal halo orbit. In Figure 2 we show the different coordinate projections of both the reference transfer trajectory and some "nearby" orbits of the stable manifold of the nominal halo orbit.

In the paper by Serban et al. ${ }^{14}$, two different strategies are considered to solve the TCM problem: the Halo Orbit Insertion (HOI) technique and the Manifold Orbit Insertion (MOI) technique. For the HOI technique, an insertion point in the halo orbit is fixed, in this way at least two manoeuvres must be done: the first one (TCM1) a few days after the departure and 

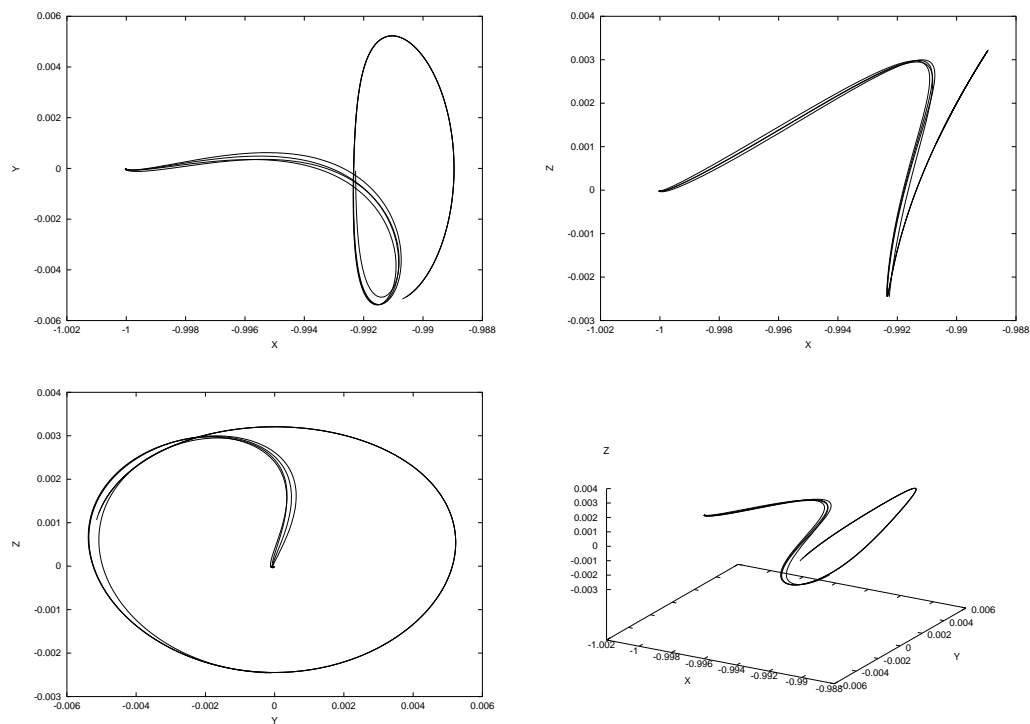

Fig. 2. Projections and 3D representation of the transfer trajectory used by Serban et $a l$. and "nearby" orbits on the stable manifold of the nominal halo orbit (RTBP units).

the last one at the HOI point. It is numerically shown that, in practice, the optimal solution can be obtained with just two TCMs, so the TCM2 is performed at the HOI point. The time of flight is not fixed in the simulations and, for the optimal costs obtained, it is found that the cost behaves almost linearly with respect to both TCM1 epoch and launch velocity error. The halo orbit insertion time is always close (with variations of the order of $20 \%$ ) to that of the reference transfer trajectory (transfer trajectory with no insertion error). For the MOI problem, the last manoeuvre is an insertion on the stable manifold of the nominal halo orbit, so there is no manoeuvre of insertion onto the halo orbit. The numerical results obtained with this approach are very close to the ones corresponding to the HOI technique. The main technical tool used through the paper is, as in the classical approach to the transfer problem, an optimisation procedure: the software package COOPT, developed at the University of Santa Barbara ${ }^{15}$. This software is used to do an optimisation of the cost function (total $\Delta v$ ) subject to the constraint of the equations of motion. In the same reference, a parametric study of the cost of the TCM is done changing and delaying the execution of the first impulse. 
In the present paper we perform the same kind of parametric study as in ${ }^{14}$ but without using any optimal control procedure. The quantitative results, concerning the optimal cost of the transfer and its behaviour as a function of the different free parameters, turn out the same. Additionally, we provide information on the cost of the transfer when the correction manoeuvres cannot be done at the optimal epochs. These results are qualitatively very close to those obtained in ${ }^{13}$ for the cost of the transfer to a Lissajous orbit around $L_{2}$, when the time of flight between de departure and the injection in the stable manifold is fixed, but the target state (position and velocity) on the manifold is varied. For this problem it is found that the cost of the transfer can rise dramatically, as will be shown also later on.

\section{The TCM1 problem for halo orbits}

\subsection{Description of the method}

We use as reference departure state, the one given in table 1 . We will always start from the fixed initial position (close to the Earth) given by this reference point. To simulate the injection error, and following ${ }^{14}$, we modify the modulus of the velocity at this initial condition according to

$$
\vec{v}(0)=\vec{v}_{\text {ref }}^{0}\left(1+\frac{\epsilon}{\left\|\vec{v}_{r e f}^{0}\right\|}\right) .
$$

where $\epsilon$ is a parameter that it is allowed to vary between $-6 \mathrm{~m} / \mathrm{s}$ and $+6 \mathrm{~m} / \mathrm{s}$, and $\vec{v}_{\text {ref }}^{0}=(-4.612683390613825,9.412034579485869$, $3.479627336419212)^{T} \mathrm{~km} / \mathrm{s}$, which, in adimensional units, correspond to the values given in table 1 . As it has already been mentioned, the departure point $\left(X_{d e p}\right)$ constructed in this way, is not on the stable manifold of the nominal halo orbit selected, but rather close to it.

The transfer path has three different legs, qualitatively represented in Figure 3:

- The first leg goes from the fixed departure point to the point where the TCM1 is performed. Usually, this correction manoeuvre takes place few days after the departure. 
- The second leg, between the two trajectory correction manoeuvres TCM1 and TCM2, is used to perform the injection in the stable manifold of the nominal orbit.

- The last path corresponds to a piece of trajectory on the stable manifold. Since both TCM1 and TCM2 are assumed to be done without errors, the spacecraft will reach the nominal halo orbit without any additional impulse.

Due to the autonomous character of the RTBP, the origin of time can be arbitrarily chosen. We assume that at the departure $t=0$. As it is explained later, we will select an "arrival point" to the halo. In this way, the TCM1, TCM2 and arrival epochs, will be denoted by $t_{1}, t_{2}$ and $t_{3}$, respectively. The values of the correction manoeuvres at $t_{1}$ and $t_{2}$ will be denoted by $\Delta v_{1}$ and $\Delta v_{2}$, respectively.

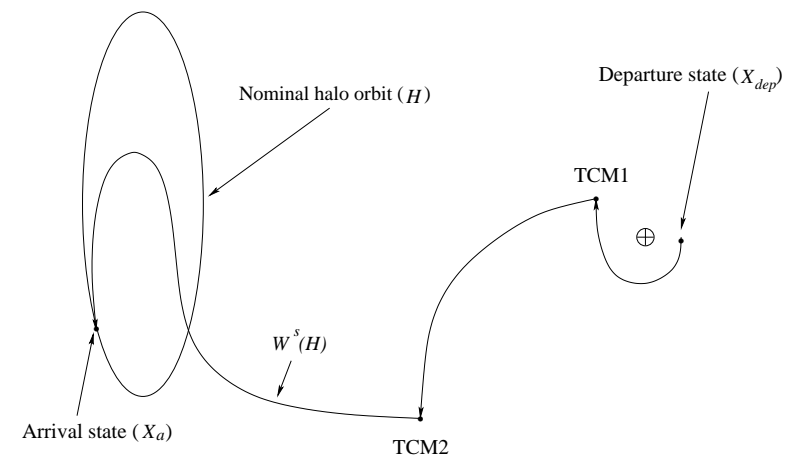

Fig. 3. The three legs used for the computation of the transfer solutions

When we say that we reach the nominal halo orbit, we mean that we are within a certain distance of a point of it, in the direction of the stable manifold. More precisely, this means that if we select a certain (short) distance, $d$, and an arrival point on the halo, $X_{a}^{h}$, the point that in fact we reach is $X_{a}=X_{a}^{h}+d \cdot V^{s}\left(X_{a}^{h}\right)$, where $V^{s}\left(X_{a}^{h}\right)$ is the linear approximation of the stable direction at the point $X_{a}^{h}$. A value of $d=200 \mathrm{~km}$ gives good results as is shown in ${ }^{4}$. We remark that the stable manifold is a two dimensional manifold (a surface in the 6-dimensional space of positions and velocities) which can be parametrised in the following way. Once a displacement $d$ has been selected, given a point $X^{h}$ on the halo orbit we 
can get an initial condition on the stable manifold $X^{h}+d \cdot V^{s}\left(X^{h}\right)$. Following the flow backwards we get all the points in the manifold associated with $X^{h}$. In this way $X^{h}$ can be thought as one of the parameters which generate the manifold. In what follows, we will call it the parameter along the orbit. The other one is the elapsed time, following the flow, from the initial condition $X_{a}=X_{a}^{h}+d \cdot V^{s}\left(X_{a}^{h}\right)$ to a certain point. We call this time interval the parameter along the flow. We remark that this parametrisation depends on the choice of $d$, a small change in $d$ produces an effect equivalent to a small change in the parameter along the orbit. This is: with a small change in $d$ we can get the same orbits of the manifold as with a small change of $X^{h}$ and only a small shift in the parameter along the orbit will be observed. This is because the stable direction is transversal to the flow.

We denote by $\phi(X, t)$ the image, under the flow of the Restricted Three Body Problem, of the point $X$ after $t$ time units. Given the departure state, $X_{d e p}$, and the time $t_{1}$, we define $X_{1}=\phi\left(X_{d e p}, t_{1}\right)$. Then, the transfer condition is stated as

$$
\phi\left(X_{1}+\Delta v_{1}, t_{2}-t_{1}\right)+\Delta v_{2}=\phi\left(X_{a}, t_{2}-t_{3}\right)
$$

where, in this relation, a term like $X_{1}+\Delta v_{1}$ has to be understood as: to the state $X_{1}$ (position and velocity) we add $\Delta v_{1}$ to the velocity. Note that for a given insertion error $\epsilon$ (which determines $X_{d e p}$ ) we have six equality constraints, corresponding to the position and velocity equations (2), and ten parameters: $t_{1}, t_{2}, t_{3}, \Delta v_{1}, \Delta v_{2}$ and $X_{a}$ (given by the parameter along the orbit) which should be chosen in an optimal way within mission constraints.

The sketch of the exploration procedure is the following. To start with, we consider $\epsilon$ and $t_{1}$ fixed. Two types of explorations appear in a natural way: the fixed time of flight transfers, for which $t_{3}$ is fixed, and the free time of flight transfers, where $t_{3}$ is allowed to vary. In both cases, we start the exploration fixing an initial value for the parameter along the orbit, $X_{a}$. In the case of fixed time of flight, the problem then reduces to seven parameters $\left(t_{2}, \Delta v_{1}, \Delta v_{2}\right)$ and the six constraints (2). Using $\Delta v_{1}$ and $\Delta v_{2}$ to match the constraints (2), the cost of the transfer, $\|\Delta v\|=\left\|\Delta v_{1}\right\|+\left\|\Delta v_{2}\right\|$, is seen as a function of $t_{2}$. In the case of free time of flight, $\|\Delta v\|$ is seen as a function of $t_{2}$ and $t_{3}$, or equivalently, as a function of $t_{2}$ and the parameter along the flow, $t_{w s}=t_{3}-t_{2}$.

Once we have explored the dependence of the transfer cost with respect 
to $t_{2}$ and $t_{3}$, we study the behaviour moving the parameter along the orbit, $X_{a}$, and finally, the dependence with respect to the parameter $\epsilon$ (which is determined by the launch vehicle) and $t_{1}$ (which, due to mission constraints, is enough to vary in a narrow and coarse range). We will see that we have some simple linear relations between them.

In order to solve equation (2) by some differential correction procedure, we need an initial guess. This is taken from the solution obtained when $\epsilon=0$. For most of the simulations, as well as for the parametric study, we use a continuation procedure to get the initial approximation of the solution. It must also be noted, that due to the strong hyperbolic behaviour of the orbits under consideration, it can be necessary to solve equation (2) using some multiple shooting method (see ${ }^{16}$ ). We could use a slight variation of the multiple shooting procedure to recover the MOI technique with more than two TCM used in ${ }^{14}$, although this possibility has not been implemented.

As a first example, Figure 4 shows the results obtained when: $\epsilon=$ $-3 \mathrm{~m} / \mathrm{s}$, the first manoeuvre is delayed 4 days after the departure $\left(t_{1}=4\right)$, the total time of flight, $t_{3}$, is taken equal to 173.25 days and the arrival point is the one given in table 2. In the next section we will come back to this Figure.

\section{Non-linear approximation of the stable manifold}

In the previous section, we have discussed how the linear approximation of the stable direction (obtained using the linearisation of the flow) can be used to globalise and parametrise the stable manifold of a periodic halo orbit. In a second approach we have used a non-linear approximation of the stable manifold. In the case of halo orbits and using the parameters mentioned in the preceding section, the results obtained with the linear approximation and the ones using the non-linear one are almost the same. Since the increase in computational cost doesn't give any extra advantage, all the computations that we present have been done using the linear approximation of the manifolds. However this non-linear study is very useful when dealing with the study of the TCM problem for Lissajous libration point trajectories, specially with big amplitudes.

Following ${ }^{2}$, in this section we summarise the procedure for the compu- 


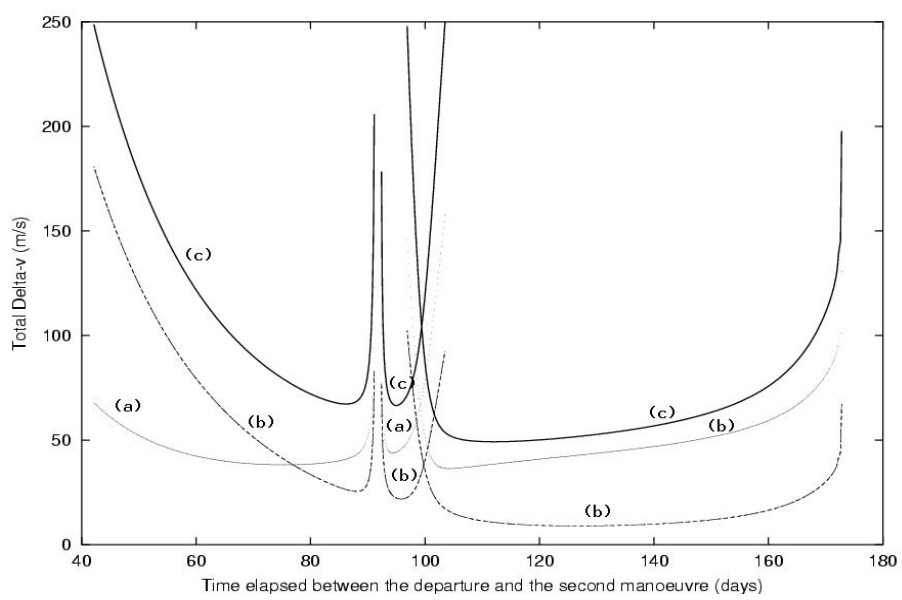

Fig. 4. Cost of the trajectory correction manoeuvres when TCM1 is delayed 4 days after departure and the total time of flight is fixed to 173.25 days. The arrival point on the nominal halo orbit is given in table 2. The curves labelled with (a) correspond to $\left\|\Delta v_{1}\right\|$, those with (b) to $\left\|\Delta v_{2}\right\|$ and those with (c) to the total cost: $\left\|\Delta v_{1}\right\|+\left\|\Delta v_{2}\right\|$.

tation of the non-linear approximation of the stable manifold for the Lissajous and halo orbits. Consider the linearised equations of the restricted three body problem around any collinear equilibrium point

$$
\begin{aligned}
\ddot{x}-2 \dot{y}-\left(1+2 c_{2}\right) x & =0, \\
\ddot{y}+2 \dot{x}+\left(c_{2}-1\right) y & =0, \\
\ddot{z}+c_{2} z & =0,
\end{aligned}
$$

where $c_{2}$ is a parameter depending on the mass ratio and the equilibrium point considered ( see $^{12}$ ). The solution of these equations is given by,

$$
\begin{aligned}
& x=\alpha_{1} e^{\lambda_{0} t}+\alpha_{2} e^{-\lambda_{0} t}+\alpha_{3} \cos \left(\omega_{0} t+\phi_{1}\right), \\
& y=\bar{k}_{2} \alpha_{1} e^{\lambda_{0} t}-\bar{k}_{2} \alpha_{1} e^{-\lambda_{0} t}+\bar{k}_{1} \alpha_{3} \sin \left(\omega_{0} t+\phi_{1}\right), \\
& z=\alpha_{4} \cos \left(\nu_{0} t+\phi_{2}\right),
\end{aligned}
$$

where $\bar{k}_{1}, \bar{k}_{2}, \omega_{0}, \nu_{0}$ and $\lambda_{0}$ are constants which can be written in terms of $c_{2}$. Finally, $\alpha_{i}$ and $\phi_{i}$ are free amplitudes and phases.

Taking $\alpha_{1}=\alpha_{2}=0$ we get libration solutions. In case that $\alpha_{1} \neq$ 0 or $\alpha_{2} \neq 0$ we get exponentially increasing or decreasing translations along privileged directions in the phase space. So, we can consider them as amplitudes in the unstable and stable directions respectively. In particular, 
setting $\alpha_{1}=0$, we get initial conditions for orbits in the stable manifold of a certain linear Lissajous orbit corresponding to the linear equations.

Using a Lindsted-Poincaré procedure we can look for a formal series solution of the nonlinear equations in terms of the four amplitudes $\alpha_{i}$ and the following three variables,

$$
\theta_{1}=\omega t+\phi_{1}, \quad \theta_{2}=\nu t+\phi_{2}, \quad \theta_{3}=\lambda t .
$$

The expansions of $x, y$ and $z$ are given by,

$$
\sum e^{(i-j) \theta_{3}}\left[\left\{\begin{array}{l}
x_{c} \\
y_{c} \\
z_{c}
\end{array}\right\}^{p q} \cos \Theta+\left\{\begin{array}{l}
x_{s} \\
y_{s} \\
z_{s}
\end{array}\right\}_{i j k m}^{p q} \sin \Theta,\right] \alpha_{1}^{i} \alpha_{2}^{j} \alpha_{3}^{k} \alpha_{4}^{m},
$$

where $\Theta=\left(p \theta_{1}+q \theta_{2}\right)$ and the summation is taken with respect to the integer index $i, j, k, m, p$ and $q$ in a suitable way. Also, according to the Lindstedt-Poincaré procedure, in order to avoid secular terms the frequencies $\omega, \nu$ and $\lambda$ must be expanded in formal power series of the four amplitudes,

$$
\begin{gathered}
\omega=\sum \omega_{i j k m} \alpha_{1}^{i} \alpha_{2}^{j} \alpha_{3}^{k} \alpha_{4}^{m}, \quad \nu=\sum \nu_{i j k m} \alpha_{1}^{i} \alpha_{2}^{j} \alpha_{3}^{k} \alpha_{4}^{m}, \\
\lambda=\sum \lambda_{i j k m} \alpha_{1}^{i} \alpha_{2}^{j} \alpha_{3}^{k} \alpha_{4}^{m},
\end{gathered}
$$

being the independent terms, $\omega_{0000}=\omega_{0}, \nu_{0000}=\nu_{0}$ and $\lambda_{0000}=\lambda_{0}$. So the expansions truncated at first order reproduce the solution of the linear equations of motion. Moreover, if we skip the terms of the expansion related with $i$ and $j$ (this is $i-j \neq 0$ ) we have expansions for Lissajous orbits but not for their invariant manifolds which turn out be the same as the ones given in ${ }^{5}$.

In the halo periodic case the procedure must be slightly modified. The solution depends only on one frequency and this fact introduces a relation between the two central amplitudes $\alpha_{3}$ and $\alpha_{4}$. The formal series expansion are given by,

$$
\left\{\begin{array}{l}
x \\
y \\
z
\end{array}\right\}=\sum e^{(i-j) \theta_{3}}\left[\left\{\begin{array}{l}
x_{c} \\
y_{c} \\
z_{c}
\end{array}\right\}_{i j k m}^{p q} \cos \left(p \theta_{1}\right)+\left\{\begin{array}{l}
x_{s} \\
y_{s} \\
z_{s}
\end{array}\right\}_{i j k m}^{p q} \sin \left(p \theta_{1}\right),\right] \alpha_{1}^{i} \alpha_{2}^{j} \alpha_{3}^{k} \alpha_{4}^{m},
$$

where again,

$$
\theta_{1}=\omega t+\phi_{1}, \quad \theta_{3}=\lambda t
$$




$$
\omega=\sum \omega_{i j k m} \alpha_{1}^{i} \alpha_{2}^{j} \alpha_{3}^{k} \alpha_{4}^{m}, \quad \lambda=\sum \lambda_{i j k m} \alpha_{1}^{i} \alpha_{2}^{j} \alpha_{3}^{k} \alpha_{4}^{m},
$$

but now one must take into account a relation between amplitudes which is given by a series expansion of the type,

$$
\sum d_{i j k m} \alpha_{1}^{i} \alpha_{2}^{j} \alpha_{3}^{k} \alpha_{4}^{m}=0 .
$$

In all these expansions there are symmetries which make many of the coefficients zero. This fact saves storage and computing time. In ${ }^{1}$ the Lissajous expansions have been tested. Using order 25 (i.e. terms up to $i+j+k+m=25$ ), differences less than $100 \mathrm{~km}$ between the numerically integrated solution and the direct evaluation of the expansion are obtained for the orbits of the manifolds up to about a distance of $500000 \mathrm{~km}$ from the Lissajous orbit.

\subsection{Fixing the arrival point and the time of flight}

For the first study of the cost of the TCM, we have taken $t_{1}=4$ days and $\epsilon=-3 \mathrm{~m} / \mathrm{s}$. For the time of flight we have used the values obtained in ${ }^{14}$ for the optimal solution, this is $t_{3}=173.25$ days. Since the arrival point is not explicitly given in the above reference, we have used the following approximation (which corresponds to integrate the reference initial state during 173.25 days)

Table 2. Approximation used for the adimensional coordinates of the arrival point, $X_{a}$, of the optimal solution.

\begin{tabular}{rrrr}
\hline$x_{a}$ & $-9.8985625832629109 E-01$ & $\dot{x}_{a}$ & $3.3913736319571984 E-03$ \\
$y_{a}$ & $4.1836615583455538 E-03$ & $\dot{y}_{a}$ & $-6.2057458211230666 E-03$ \\
$z_{a}$ & $2.1771475345925264 E-03$ & $\dot{z}_{a}$ & $4.1484980583161675 E-03$ \\
\hline
\end{tabular}

As it has already been said, with the values of these parameters fixed, we get a one dimensional set of possibilities, which are the ones displayed in Figure 4. In the Figure, we show the cost of the two Trajectory Correction Manoeuvres, as well as the total cost, in front of the epoch of execution of the second manoeuvre, $t_{2}$. Several remarks should be done in connection with the Figure: 
- The solutions of equation (2) are grouped along, at least, three curves. For $t_{2}=99.5$ days there is a double point in the cost function, corresponding to two different possibilities. In Figure 5 we have represented both as well as the orbit of the stable manifold where we perform the injection. The qualitative behaviour of both solutions is rather different.
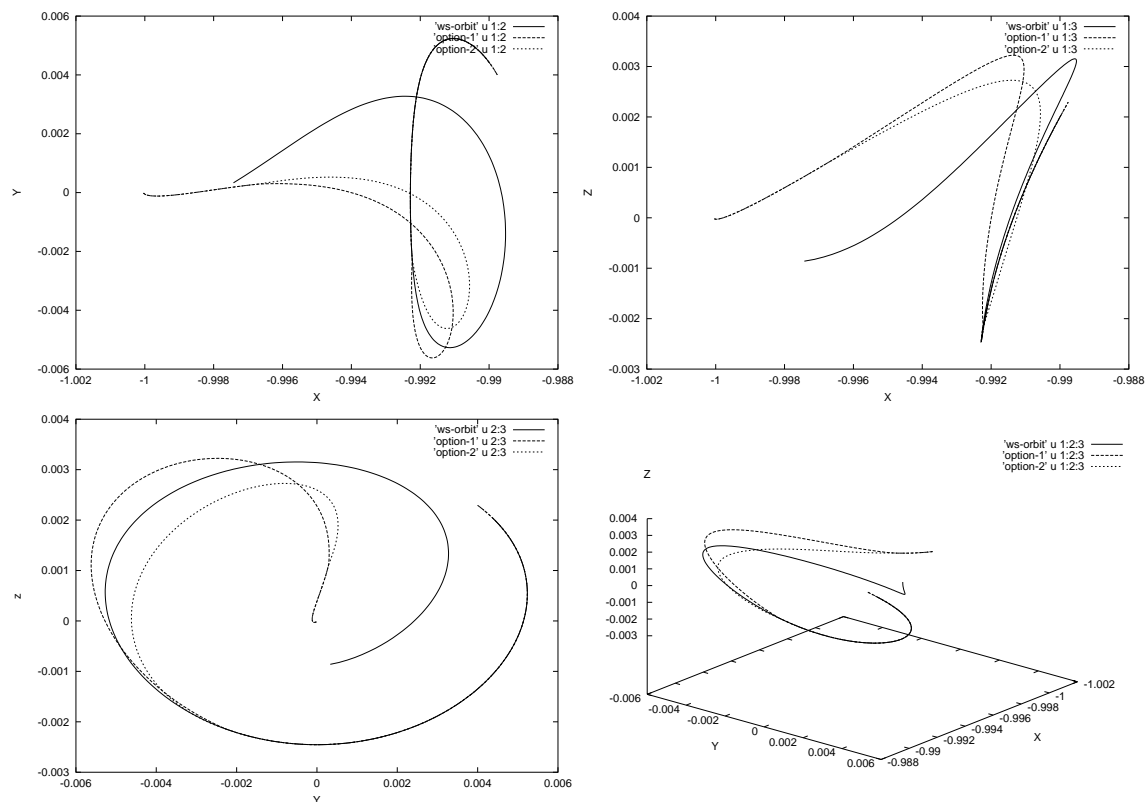

Fig. 5. Coordinate projections and 3D representation of the two solutions obtained for $t_{2}=99.5$ days (double point of the cost function). In the Figures we have represented also the orbit of the stable manifold of the nominal orbit where we perform the injection.

- For $t_{2}=113$ days we get the optimum solution in terms of fuel consumption: $\left\|\Delta v_{1}\right\|+\left\|\Delta v_{2}\right\|=49.31 \mathrm{~m} / \mathrm{s}$. This value is very close to the one given in ${ }^{14}$ for the MOI approach, which is $49.1817 \mathrm{~m} / \mathrm{s}$. The discrepancies can be attributed to slight differences between the two nominal orbits and the corresponding target points.

- When $t_{2}$ is small or very close to the final time, $t_{3}$, the total cost of the TCMs increases, as it should be expected.

- Around the values $t_{2}=92,97$ and 102 days, the total cost increases 


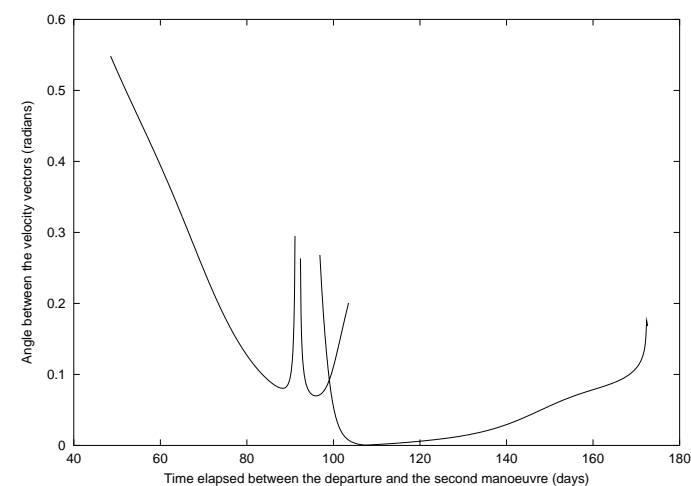

Fig. 6. Using the values of the parameters to get Figure 4, here we represent the angle (in radians) between the two velocity vectors, the ones just prior and after the TCM2 epoch.

abruptly. This sudden grow is analogous to the one described in ${ }^{8}$ in connection with the TCM problem for the Genesis mission. It is also similar to the behaviour found in ${ }^{13}$ for the cost of the transfer to a Lissajous orbit around $L_{2}$, when the time of flight between the departure and the injection in the stable manifold is fixed. To explain this fact, we have computed the angle between the two velocity vectors at $t=t_{2}$, this is when changing from the second to the third leg of the transfer path. This angle has been represented in Figure 6, and looking at it we see that it also increases sharply at the corresponding epochs. This seams to be the geometrical reason for the detected behaviour.

As a second step, we have done a first parametric study allowing variations in the epoch of the execution of TCM1, $t_{1}$, and in $\epsilon$. Partial results are given in table 3 . In the last column of this table we include the numerical results obtained by Serban et al. $\left({ }^{14}\right)$ for the MOI strategy (those corresponding to HOI trajectories are similar), which are very close to ours. From this table, it is clearly seen that the behaviour of the optimal cost with respect to $\epsilon$ is linear. In Figure 7 we represent the results corresponding to a larger set of explorations, where we allow variations in the magnitude of the error, $\epsilon$, and in the epoch $t_{1}$. From it, it is also clear a linear behaviour of the optimal cost with respect to $t_{1}$.

In the next step of our study we allow variations in the parameter along the orbit. Assuming the periodic halo orbit parametrised by time (the pe- 
Table 3. Numerical results of the parametric study of the TCM cost. The simulations have been done fixing the arrival point as in table 2 and the total time of flight $t_{3}=173.25$ days in order to compare the results with the ones obtained by Serban et al. which are displayed in the last column.

\begin{tabular}{|c|c|c|c|c|c|}
\hline$t_{1}$ & $\epsilon$ & $\left|\Delta v_{1}\right|$ & $\Delta v_{2} \mid$ & $\left|\Delta v_{1}\|+\| \Delta v_{2}\right| \mid$ & $\Delta v_{o p t}\left({ }^{14}\right)$ \\
\hline 3 & 5 & 51.4551 & 15.2748 & 66.7299 & \\
\hline 3 & 4 & 41.2822 & 14.7060 & 55.9882 & \\
\hline 3 & 3 & 31.0891 & 14.1944 & 45.2835 & \\
\hline 3 & 2 & 20.8642 & 13.7963 & 34.6605 & \\
\hline 3 & 1 & 10.5610 & 13.6390 & 24.2000 & \\
\hline 3 & 0 & 0.0974 & 14.0324 & 14.1298 & \\
\hline 3 & -1 & 10.8051 & 13.4634 & 24.2685 & \\
\hline 3 & -2 & 21.7421 & 12.8390 & 34.5811 & \\
\hline 3 & -3 & 32.7661 & 12.2004 & 44.9665 & 45.1427 \\
\hline 3 & -4 & 43.8878 & 11.5459 & 55.4337 & 55.6387 \\
\hline 3 & -5 & 55.1127 & 10.8796 & 65.9923 & 65.9416 \\
\hline 4 & 5 & 59.0547 & 15.2508 & 74.3055 & \\
\hline 4 & 4 & 47.4145 & 14.5247 & 61.9392 & \\
\hline 4 & 3 & 35.7505 & 13.8649 & 49.6154 & \\
\hline 4 & 2 & 24.0530 & 13.3523 & 37.4053 & \\
\hline 4 & 1 & 12.2530 & 13.1986 & 25.4516 & \\
\hline 4 & 0 & 0.0987 & 14.0261 & 14.1248 & \\
\hline 4 & -1 & 12.7285 & 12.9531 & 25.6816 & \\
\hline 4 & -2 & 25.5010 & 11.9398 & 37.4408 & \\
\hline 4 & -3 & 38.3502 & 10.9663 & 49.3165 & 49.1817 \\
\hline 4 & -4 & 51.3480 & 9.8576 & 61.2056 & 61.5221 \\
\hline 4 & -5 & 64.5745 & 8.9561 & 73.5306 & 73.4862 \\
\hline 5 & 5 & 66.1938 & 15.2117 & 81.4055 & \\
\hline 5 & 4 & 53.1763 & 14.3203 & 67.4966 & \\
\hline 5 & 3 & 40.1315 & 13.4972 & 53.6287 & \\
\hline 5 & 2 & 27.0602 & 12.8413 & 39.9015 & \\
\hline 5 & 1 & 13.8820 & 12.6547 & 26.5367 & \\
\hline 5 & 0 & 0.1172 & 14.0080 & 14.1252 & \\
\hline 5 & -1 & 14.6289 & 12.3175 & 26.9464 & \\
\hline 5 & -2 & 29.1862 & 10.8579 & 40.0441 & \\
\hline 5 & -3 & 43.8275 & 9.5245 & 53.3520 & 53.9072 \\
\hline 5 & -4 & 58.8400 & 8.1134 & 66.9534 & 66.8668 \\
\hline 5 & -5 & 74.2527 & 6.6145 & 80.8672 & 81.1679 \\
\hline
\end{tabular}

riod of the orbit is approximately equal to 180 days) we have taken a total number of "arrival points" equal to 36, evenly spaced in time. In Figure 8 we show the behaviour of the total cost of the trajectory correction manoeuvres when the parameter along the orbit is changed around the value corresponding to the optimal solution (which is also displayed in the Figure). In the left plot the displayed curves correspond to adding 5, 10 and 15 days respectively, to the parameter along the orbit and the one in the right hand side to decrease this parameter in 5 and 10 days. We represent only 


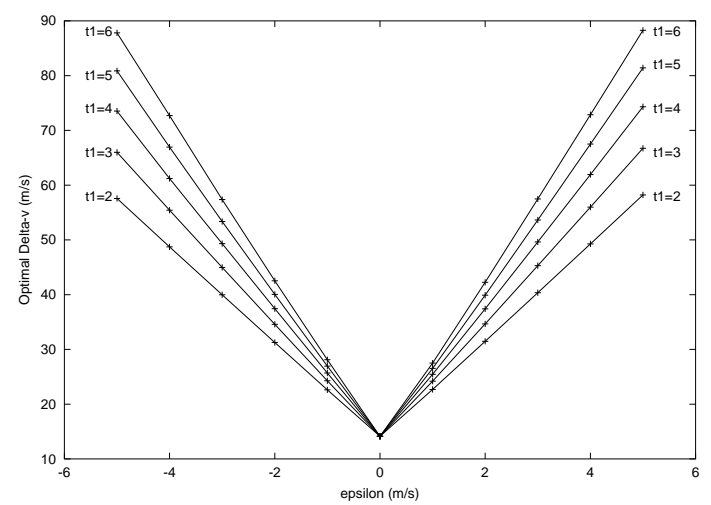

Fig. 7. Behaviour of the optimal cost vs $\epsilon$ for different values of $t_{1}$

TCM with a total $\|\Delta v\|=\left\|\Delta v_{1}\right\|+\left\|\Delta v_{2}\right\|$ smaller than $300 \mathrm{~m} / \mathrm{s}$. Increasing o decreasing the values of the parameter along the orbit out of the range of the ones represented in the figures, the total cost increases, and the results obtained are always over the threshold fixed for the representation. This is also the reason because one of the three pieces of the optimal solution has disappeared from the plots. In Figure 9 we plot the surface representing the cost when changing the parameter along the orbit (the value 0 of this parameter corresponds to the point $X_{a}$ given in table 2). Since the total time of flight has been fixed, we get only total TCM costs below $300 \mathrm{~m} / \mathrm{s}$ within the ranges displayed in the figures.
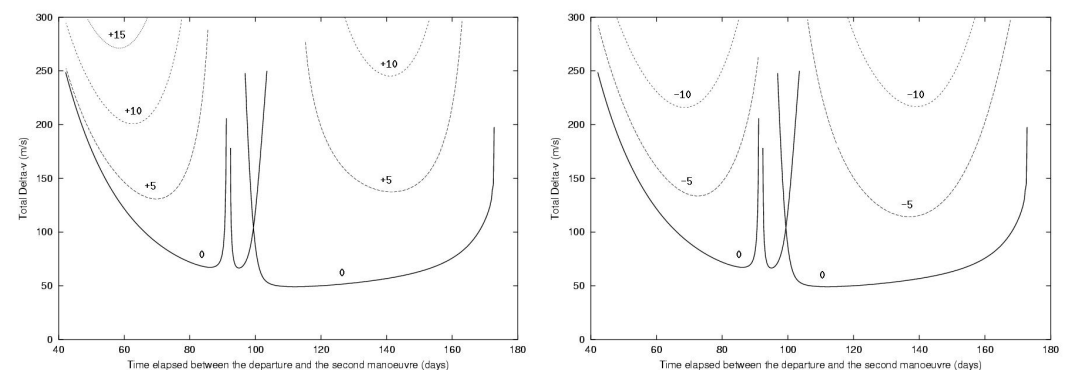

Fig. 8. Total cost of the trajectory correction manoeuvres when the "arrival" point at the halo orbit is changed. Numerical results correspond to changing the arrival point by adding (left figure) or subtracting (right figure) 5, 10 and 15 days to the parameter along the orbit corresponding to the optimal solution which is represented by the lowest curve. 

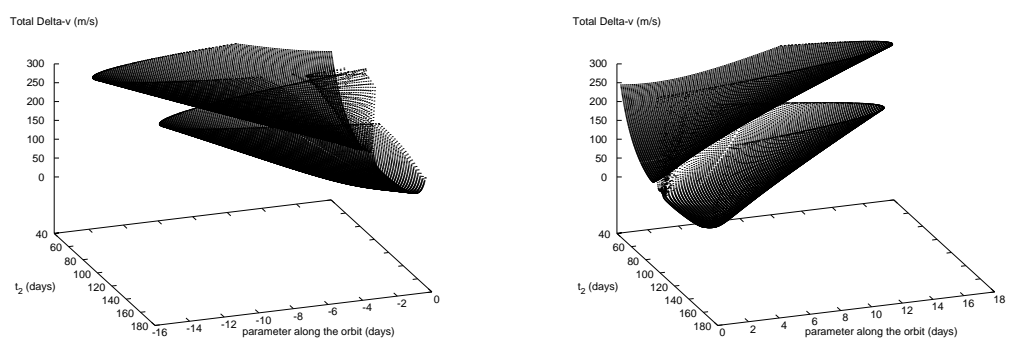

Fig. 9. Total cost of the trajectory correction manoeuvres when the "arrival" point, represented by the parameter along the orbit, is moved around the point $X_{a}$ given in table 2, the total time of flight is fixed to 173.25 days and the first manoeuvre is delayed 4 days after the departure. We display the results for negative and positive variations of the parameter along the orbit on the left and right-hand side figures, respectively.

\subsection{Free time of flight}

To start with, we take $t_{1}=4$ days, $\epsilon=-3 \mathrm{~m} / \mathrm{s}$ and the arrival point of the preceding sections. With all these parameters fixed, the transfer condition (2) has a two dimensional set of solutions, which can be parametrised by $t_{2}$ and the parameter along the flow, $t_{w s}=t_{3}-t_{2}$, which give the insertion point into the stable manifold. In Figure 10 we show some sections of this surface, for different values of the parameter along the flow, $t_{w s}$ ranging from 40 days (right curve) to 125 days (left curve) as well as the solution that we have obtained in the preceding section for $t_{3}=173.25$ days. Several remarks should be done with respect to this figure:

- There are values of $t_{2}$ and $t_{w s}$ (for instance $t_{2}=108.125, t_{w s}=65$ days) for which the total cost is less than the values we have obtain for $t_{3}=173.25$ days.

- If we take into consideration that the curves that we have plotted in Figure 10 correspond to evenly spaced values of $t_{w s}$, it seems that the value of $t_{2}$ that makes the cost optimal is a linear function of $t_{w s}$, at least in the right hand side of the figure where we are close to the optimum values $\left(t_{2}>100\right.$; the curves in this region correspond to values of $t_{w s}$ equal to $70,65,60,55,50,45$ and 40 days). Assuming $t_{2}=m\left(t_{w s}-t_{w s}^{0}\right)+t_{2}^{0}$ the value of $m$ is close to minus one, since the couple $\left(t_{2}, t_{w s}\right)$ that makes minimum the cost verifies $t_{w s}+t_{2} \simeq 173.3$ days. This fact justifies why the cost function we obtain for $t_{3}=173.25$ days is very close to the optimal solution for $t_{3}$ free. 


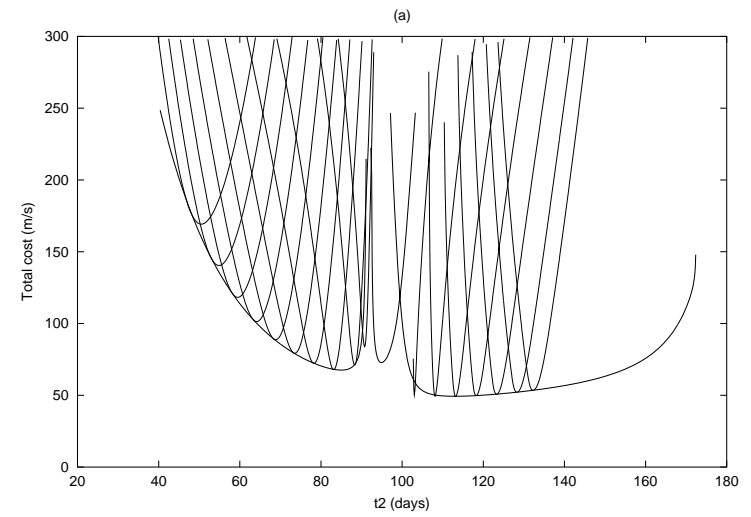

Fig. 10. The curves appearing in the figure are slices of the surface representing the minimum total cost of the TCM, for different values of $t_{w s}$. In the computations, the first manoeuvre has been delayed 4 days after the departure, the total time of flight is free and the arrival point is fixed. The lowest curve, which almost envelopes the different slices, is the cost function when the total time of flight is fixed to 173.25 days.

To study the influence of the variations in the parameter along the orbit, which is equivalent to change the arrival point, we have taken 12 arrival points evenly spaced in time, displayed in Figure 11. In Figure 12(a) we show the behaviour of the optimal cost for the first six values of the parameter along the orbit. After point number six, the cost function increases sharply and we have not represented the results associated to them. We see that in the region between the $4^{\text {th }}$ and $5^{\text {th }}$ point there is an optimal solution. Taking values of the parameter along the orbit between these two ones, in Figure 12(b) we show the curves of minimum cost as a function of $t_{w s}$. Each curve corresponds to a different value of the parameter along the orbit varying between 0.8 and 1.6 (with step 0.1 ).

As before, it is interesting to observe that the values of $t_{2}$ and $t_{w s}$ that minimise the total cost behave linearly, with respect to the parameter along the orbit, when we are near to the optimal solution. This is shown in Figures 12(c) and 12(d). Using this fact we have obtained that the optimal solution corresponds to $t_{h}=61.34$ days with a total cost of $49.1861 \mathrm{~m} / \mathrm{s}$. The insertion manoeuvre takes place 111.14 days after the departure with a total time of flight of 172.27 days. This optimal solution is displayed in Figure 13. 


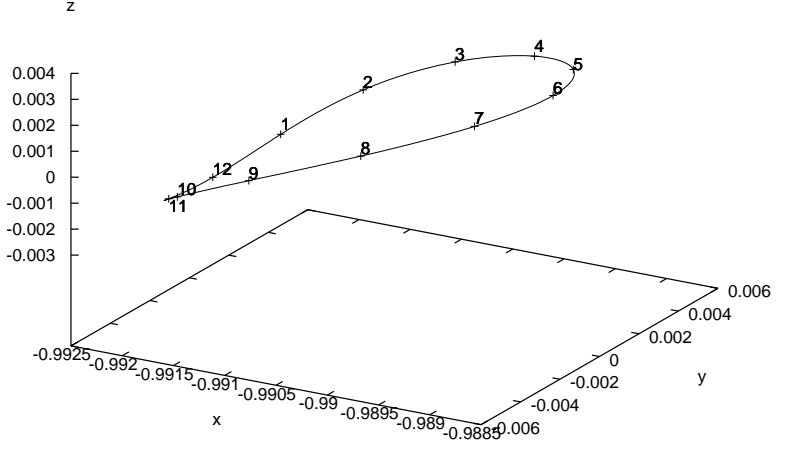

Fig. 11. 3D representation of the nominal halo orbit and the 12 "arrival points", evenly spaced in time, that have been used in our simulation.

As a final exploration we allow variations in the size of the target halo orbit. We have done the computations using halo orbits with values of the z-amplitude $\beta$ equal to 0.08 and 0.18 in addition to the value 0.28 used in the preceding simulations. In table 4 we give the results obtained using the same nominal departure point for all of them. We remark that when the amplitude of the nominal orbit decreases, the total cost of the optimal TCM increases as well as the value of the parameter along the flow $\left(t_{w s}\right)$ which corresponds to the optimal solution.

Table 4. Optimal solution for different normalised $\mathrm{z}$-amplitude $(\beta)$ halo orbits. The departure point has been taken as in table 1 .

\begin{tabular}{crrr}
\hline$\beta$ & 0.28 & 0.18 & 0.08 \\
$t_{2}$ (days) & 111.14 & 146.61 & 69.70 \\
$t_{w s}($ days $)$ & 61.13 & 111.55 & 156.80 \\
Cost $(\mathrm{m} / \mathrm{s})$ & 49.1861 & 97.0549 & 170.9650 \\
\hline
\end{tabular}

\section{Departing from the stable manifold}

In this section we show the results corresponding to take the departure point on the stable manifold of the target orbit. Now, instead of using the 

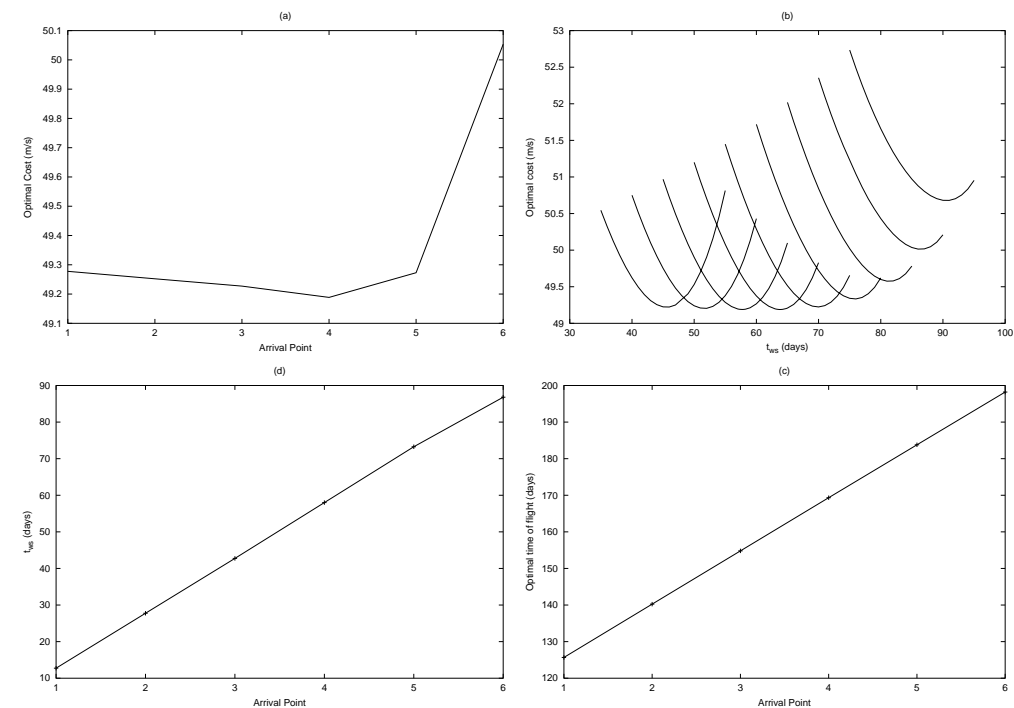

Fig. 12. (a) Minimum total cost of the trajectory correction manoeuvres when the first manoeuvre is delayed 4 days, $\epsilon=-3 \mathrm{~m} / \mathrm{s}$, the total time of flight is free and the arrival point is varied. (b) Section of the cost surface for different values of the parameter along the orbit between the $4^{t h}$ and $5^{t h}$ arrival point. (c) Optimal time between the insertion into the stable manifold and the arrival when the arrival point is varied. (d) Optimal time of flight when the arrival point is varied.

departure conditions given in table 1 , we take as initial position and velocity a point on the stable manifold, with the velocity components affected by some error. If the error is set equal to zero, then no TCM is needed to reach the target orbit.

We use the same nominal halo orbit of the preceding sections, this is: a halo orbit around the $L_{1}$ point of the Earth-Sun system, with normalised $z$-amplitude $\beta=0.28$. Taking the parameter along the orbit between $[0,2 \pi]$, in Figure 14 we represent the minimum distance to the Earth of the stable manifold of the nominal orbit at its first close passage following the parameter along the flow. As it can be seen, there are orbits which collide with the Earth (their minimum distance is below its equatorial radius). We have selected for the departure point one on the orbit associated to a value of the parameter along the orbit equal to $t_{h}=3.66000$ and at a distance from the centre of the Earth equal to $6578 \mathrm{~km}$; the adimensional coordinates of this point are given in table 5 and, as it can be seen, are not too far from those given in table 1 . 

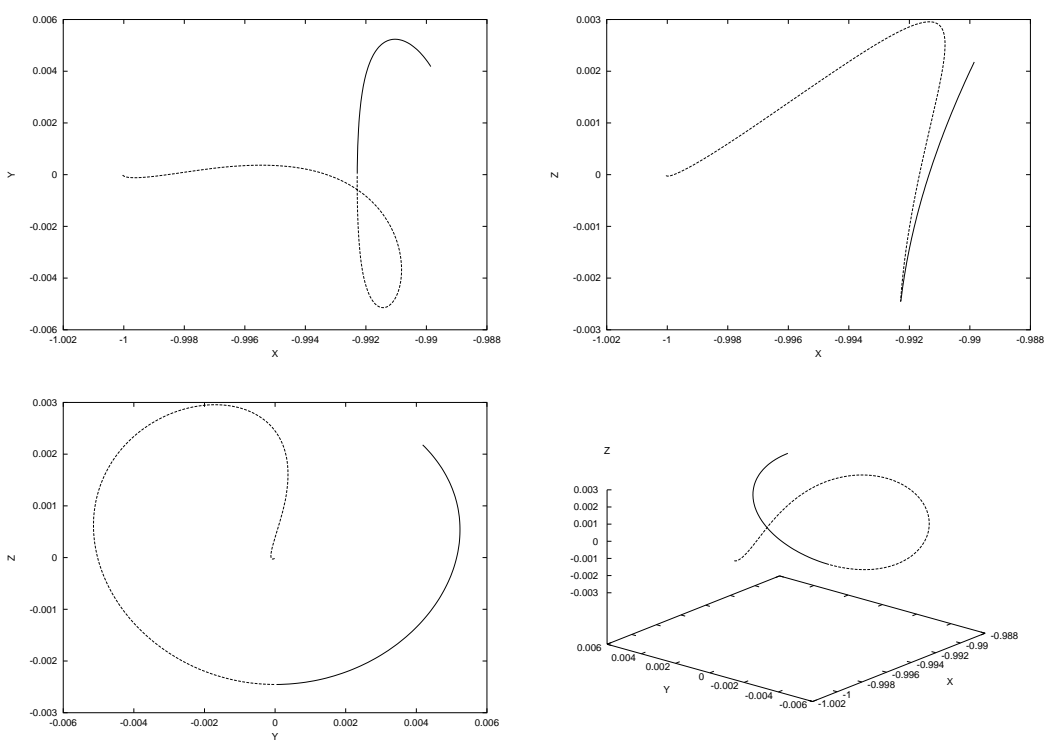

Fig. 13. Projections and 3D representation of the optimal transfer trajectory with $t_{2}$ $=111.14$ days, $t_{w s}=61.13$ days and total $\delta v=49.1861 \mathrm{~m} / \mathrm{s}$.
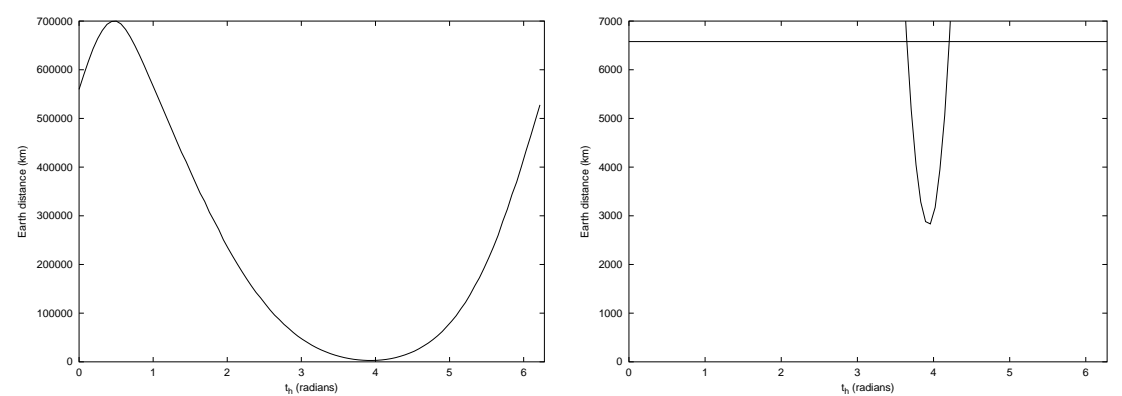

Fig. 14. Minimum distance to the Earth of the orbits of the stable manifold of the nominal halo orbit with $\beta=0.28$. The distance is below $6578 \mathrm{~km}$ for the values of the parameter along the orbit (with values in $[0,2 \pi]$ ) $t_{h}$ between 3.648511 and 4.207157 as can be seen in the magnification.

Now, adding $\epsilon=7 \mathrm{~m} / \mathrm{s}$ to the three velocity components of the nominal point given in table 5 , we compute the departure point which will be used for the explorations (the parametric study varying the value of $\epsilon$ gives results qualitatively analogous to the ones already described). As time of flight we 
Table 5. Adimensional coordinates of the nominal departure point on the stable manifold.

\begin{tabular}{lllr}
\hline$x_{0}^{\text {nom }}$ & $-1.000036453220198 E+00$ & $\dot{x}_{0}^{\text {nom }}$ & $1.618213815598005 E-01$ \\
$y_{0}^{\text {nom }}$ & $-9.466006191933124 E-06$ & $\dot{y}_{0}^{\text {nom }}$ & $-2.526026481278061 E-01$ \\
$z_{0}^{\text {nom }}$ & $-1.128673413649424 E-05$ & $\dot{z}_{0}^{\text {nom }}$ & $-2.308399055627169 E-01$ \\
\hline
\end{tabular}

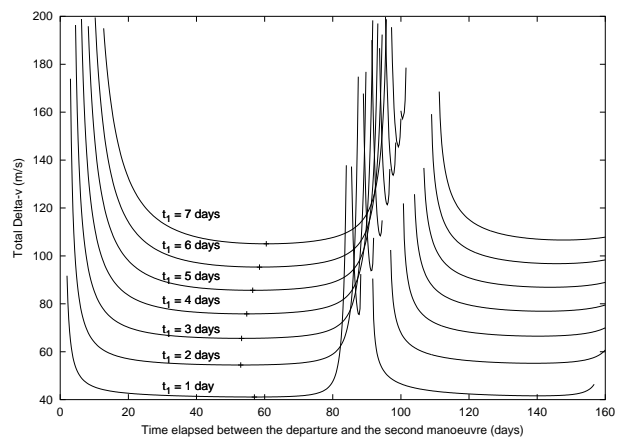

Fig. 15. Total cost of the TCM, as a function of $t_{2}$, for different values of $t_{1}$. The points with a cross on each curve correspond to the optimum cost

take the value $t_{3}=217.28$, which is the total time required by the orbit with the initial conditions given in table 5 to reach the arrival point, $X_{a}$, at the halo orbit (always at a distance of $200 \mathrm{~km}$, in the direction of the stable manifold). In Figure 15 we show the total cost (in $\mathrm{m} / \mathrm{s}$ ) of the TCM as a function of $t_{2}$, for different values of $t_{1}$ between 1 and 7 days. On each curve we have marked with a cross the points corresponding to the minimum cost. From this figure one clearly sees that:

(1) As $t_{1}$ increases, the cost of TCMs also does, and it behaves almost linearly with respect to $t_{1}$ in the selected range.

(2) The cost of the TCMs is about a $20 \%$ less than the values given in table 3 , when the departure point is not taken on the stable manifold.

(3) The optimal values of $t_{2}$ move around $t_{2}=58$ days, and approximately after 82 days $\left(t_{2}=140\right.$ days) one finds also values for TCM1 very close to the optimal ones.

As a final exploration we allow variations of the insertion point along the stable manifold. In particular if we fix $t_{1}=1$ day and $t_{2}=57$ days, we get a target insertion point on the stable manifold, which corresponds 


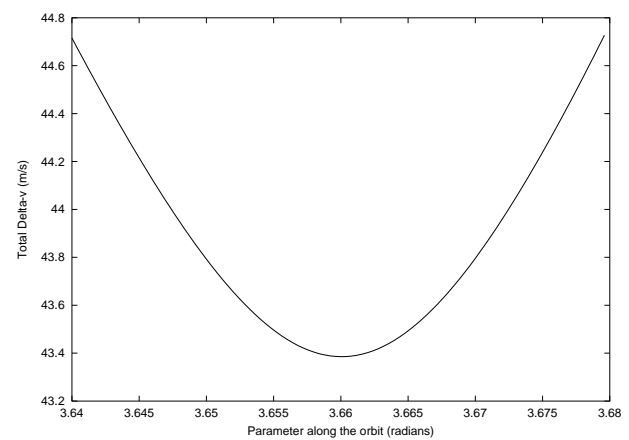

Fig. 16. Total cost of the TCM for different values of the parameter along the orbit.

to a parameter along the orbit approximately equal to 3.66. Now, we have allowed values of the parameter along the orbit between 3.64 and 3.68 and we have studied the total cost of the TCM, keeping fixed the values of $t_{1}$ and $t_{2}$, and taking as insertion point the one at minimum distance to the target point of insertion already described. The results are given in Figure 16 , from which we see that the cost increases when we move away from the most "natural" trajectory.

\section{Conclusions}

(1) The TCM problem can be studied just by using simple Dynamical Systems concepts.

(2) For the optimal TCM, the results obtained with this approach agree, qualitatively and quantitatively, with those obtained with the help of optimal control software.

(3) For periodic halo orbits, the use of the linear approximation of the stable manifold gives the same results as the non linear one.

(4) The developed procedure can be used for any kind of libration point orbit.

\section{Acknowledgements}

The research of G.G. and J.M. has been partially supported by grants DGICYT BFM2000-805 (Spain) and CIRIT 2000 SGR-27 (Catalonia). M.M. 
wishes to acknowledge the support of the doctoral research grant AP20013064 from the Spanish Ministerio de Educación, Cultura y Deportes.

\section{References}

1. J. Cobos and M. Hechler. FIRST/PLANCK Mission Analysis: Transfer to a Lissajous Orbit Using the Stable Manifold. Technical Report MAS Working Paper No. 412, ESOC, 1998.

2. J. Cobos and J.J. Masdemont. Transfers between Lissajous Libration Point Orbits. Technical report, In preparation.

3. R.W. Farquhar. The Control and Use of Libration Point Satellites. Technical Report TR R346, Stanford University Report SUDAAR-350 (1968). Reprinted as NASA, 1970.

4. G. Gómez, À. Jorba, J. Masdemont, and C. Simó. Study of the Transfer from the Earth to a Halo Orbit Around the Equilibrium Point $L_{1}$. Celestial Mechanics, 56(4):541-562, 1993.

5. G. Gómez, À. Jorba, J.J. Masdemont, and C. Simó. Dynamics and Mission Design Near Libration Point Orbits - Volume 3: Advanced Methods for Collinear Points. World Scientific, 2001.

6. G. Gómez, J. Llibre, R. Martínez, and C. Simó. Dynamics and Mission Design Near Libration Point Orbits - Volume 1: Fundamentals: The Case of Collinear Libration Points. World Scientific, 2001.

7. M. Hechler. SOHO Mission Analysis $L_{1}$ Transfer Trajectory. Technical Report MAO Working Paper No. 202, ESA, 1984.

8. K.C. Howell and B.T. Barden. Brief Summary of Alternative Targeting Strategies for TCM1, TCM2 and TCM3. Private communication. Purdue University, 1999.

9. J.A. Kechichian. The Efficient Computation of Transfer Trajectories Between Earth Orbit and $L_{1}$ Halo Orbit within the Framework of the Sun-Earth Restricted Three Body Problem. In AAS/AIAA Space Flight Mechanics Meeting, Clearwater, Florida, USA, AAS Paper 00-174, 2000.

10. M.W. Lo, B.G. Williams, W.E. Bollman, D. Han, Y. Hahn, J.L. Bell, E.A. Hirst, R.A. Corwin, P.E. Hong, K.C. Howell, B.T. Barden, and R.S. Wilson. Genesis Mission Design. In AIAA Space Flight Mechanics, Paper No. AIAA 98-4468, 1998.

11. D.L. Mains. Transfer Trajectories from Earth Parking Orbits to $L_{1}$ Halo Orbits. Master's thesis, Department of Aeronautics and Astronautics, Purdue University, Purdue, USA, 1993.

12. D.L. Richardson. Analytical Construction of Periodic Orbits About the Collinear Points. Celestial Mechanics, 22(3):241-253, 1980.

13. R.S. Wilson, K.C. Howell and M.W. Lo. Optimization of Insertion Cost Transfer Trajectories to Libration Point Orbits. Advances in the Astronautical Sciences, 103:1569-1586, 2000. 
14. R. Serban, W.S. Koon, M.W. Lo, J.E. Marsden, L.R. Petzold, S.D. Ross, and R.S. Wilson. Halo Orbit Mission Correction Maneuvers Using Optimal Control. Automatica, 38:571-583, 2002.

15. R. Serban and L.R. Petzold. COOPT - A Software Package for Optimal Control of Large-Scale Differential-Algebraic Equation Systems. Journal of Mathematics and Computers in Simulation, 56(2):187-203, 2001.

16. J. Stoer and R. Bulirsch. Introduction to Numerical Analysis. Springer Verlag, 1983. 